\title{
Visitor log for the Oregon State Arthropod Collection, January-December, 2018.
}

The Oregon State Arthropod Collection is generally open for research-related visits during the normal business hours of Oregon State University. Numerous non-research visits are also scheduled throughout the year. The collection strives to record this usage of the collection in our visitor log, a physical sign-in book located near the entrance of the collection. The table below records those entries for the 2018 calendar year, although a few illegible entries were necessarily omitted. A scanned pdf of the pages themselves may be added at a later date and if so, will be added as a 'supplemental file' to this publication.

Visitors wishing to cite their visit to the collection in scholarly work may do so using the following citation:

Oregon State Arthropod Collection. 2019. Visitor log. January-December, 2018. Catalog: Oregon State Arthropod Collection 3(1). pp 7-9. http:/ / dx.doi.org/10.5399/osu/cat osac.3.1.4563

\begin{tabular}{|c|c|c|c|c|c|c|c|}
\hline date & name & affiliation & $\begin{array}{l}\text { city/state/ } \\
\text { country }\end{array}$ & $\begin{array}{l}\text { purpose of } \\
\text { visit }\end{array}$ & taxon & visit category & research desc. \\
\hline 3-Jan & James LaBonte & $\begin{array}{l}\text { Oregon } \\
\text { Department } \\
\text { of } \\
\text { Agriculture }\end{array}$ & Salem OR & specimen use & Coleoptera: Carabidae & research & faunistics \\
\hline 4-Jan & Ron Lyons & & Coos Bay, OR & $\begin{array}{l}\text { specimen use } \\
\text { | library use }\end{array}$ & & research & faunistics \\
\hline 18-Jan & Ron Lyons & & Coos Bay, OR & specimen use & $\begin{array}{l}\text { Coleoptera: } \\
\text { Chrysomelidae: } \\
\text { Galerucinae } \\
\end{array}$ & research & faunistics \\
\hline 23-Jan & Jenny Doh & & South Korea & visit & & $\begin{array}{l}\text { professional } \\
\text { connections }\end{array}$ & \\
\hline 23-Jan & Yabin Song & & South Korea & visit & & $\begin{array}{l}\text { professional } \\
\text { connections }\end{array}$ & \\
\hline 24-Jan & Ron Lyons & & Coos Bay, OR & specimen use & $\begin{array}{l}\text { Coleoptera: } \\
\text { Chrysomelidae: } \\
\text { Galerucinae } \\
\end{array}$ & research & faunistics \\
\hline 26-Jan & $\begin{array}{l}\text { Jonathan, Christine } \\
\text { Ellingson and son } \\
\text { Nikolaj }\end{array}$ & & Dallas, OR & $\begin{array}{l}\text { visit to show } \\
\text { seven year old } \\
\text { son museum } \\
\end{array}$ & & outreach & \\
\hline 1-Feb & Ron Lyons & & Coos Bay, OR & specimen use & $\begin{array}{l}\text { Coleoptera: } \\
\text { Chrysomelidae: } \\
\text { Galerucinae } \\
\end{array}$ & research & faunistics \\
\hline 22-Feb & Peter Landolt & & & specimen use & $\begin{array}{l}\text { Hymenoptera: Sphecidae } \\
\text { I Lepidoptera: Arctiidae }\end{array}$ & $\begin{array}{l}\text { research \& } \\
\text { curation }\end{array}$ & \\
\hline 23-Feb & James LaBonte & & Salem OR & specimen use & $\begin{array}{l}\text { Coleoptera: Curculionidae } \\
\text { Scolytinae }\end{array}$ & research & taxonomy \\
\hline 28-Feb & Ron Lyons & & Coos Bay, OR & specimen use & Diptera: Tachinidae & research & diagnostics \\
\hline 8-Mar & School Group & $\begin{array}{l}\text { Anchor } \\
\text { Academy }\end{array}$ & Springfield, OR & $\begin{array}{l}\text { fieldtrip - } \\
\text { Arthropod } \\
\text { Diversity } \\
\end{array}$ & & outreach & \\
\hline 29-Mar & Jan Peters & $\begin{array}{l}\text { Florida } \\
\text { A\&M } \\
\text { University }\end{array}$ & Tallahasee, FL & specimen use & Ephemeroptera: fossils & research & taxonomy \\
\hline 29-Mar & $\begin{array}{l}\text { Abby, Ada \& Harold } \\
\text { Krueger }\end{array}$ & & & visit & & outreach & \\
\hline
\end{tabular}




\begin{tabular}{|c|c|c|c|c|c|c|c|}
\hline date & name & affiliation & $\begin{array}{l}\text { city } / \text { state/ } \\
\text { country }\end{array}$ & $\begin{array}{l}\text { purpose of } \\
\text { visit }\end{array}$ & taxon & visit category & research desc. \\
\hline 4 -Feb & Lincoln Best & & & specimen use & $\begin{array}{l}\text { Hymenoptera: } \\
\text { Megachilidae }\end{array}$ & curation & \\
\hline 11-Apr & James Miskelly & $\begin{array}{l}\text { Royal BC } \\
\text { Museum }\end{array}$ & $\begin{array}{l}\text { Vancouver, BC, } \\
\text { Canada }\end{array}$ & specimen use & Orthoptera & research & taxonomy \\
\hline 13-Apr & Ron Lyons & & Coos Bay, OR & specimen use & Orthoptera: Tridactylidae & research & faunistics \\
\hline 26-Apr & Richard D. Sage & & & $\begin{array}{l}\text { museum/ } \\
\text { collection } \\
\text { consultation }\end{array}$ & & $\begin{array}{l}\text { professional } \\
\text { connections }\end{array}$ & \\
\hline 21-May & Ron Lyons & & Coos Bay, OR & specimen use & $\begin{array}{l}\text { Coleoptera: } \\
\text { Chrysomelidae: } \\
\text { Galerucinae }\end{array}$ & research & faunistics \\
\hline 6-Jul & Scott Fitzgerald & & Corvallis, OR & specimen use & Diptera & research & taxonomy \\
\hline 9-Jul & Bruce Ohara & & & specimen use & Lepidoptera & research & faunistics \\
\hline 30-Jul & Loren Russell & & Corvallis, OR & specimen use & Orthoptera: Tridactylidae & research & \\
\hline 7-Aug & David Wagner & $\begin{array}{l}\text { University } \\
\text { of } \\
\text { Connecticutt }\end{array}$ & Storrs, CT & specimen use & Lepidoptera: Hepialidae & research & taxonomy \\
\hline 10-Aug & Ron Lyons & & Coos Bay, OR & specimen use & $\begin{array}{l}\text { Coleoptera: } \\
\text { Chrysomelidae: } \\
\text { Galerucinae }\end{array}$ & research & faunistics \\
\hline 17-Aug & Ron Lyons & & Coos Bay, OR & specimen use & Coleoptera- coastal fauna & research & faunistics \\
\hline 23-Aug & Sarah Gardener & & Pendleton, OR & specimen use & Hymenoptera: Apoidea & research & diagnostics \\
\hline 6-Sep & Philip Nosler & & Damascus, OR & specimen use & Diplopoda & research & taxonomy \\
\hline 11-Sep & $\begin{array}{l}\text { Eleanor Des Jardins \& } \\
\text { Irene Isley }\end{array}$ & & & visit & & outreach & \\
\hline 28-Sep & Will Jesse & & & specimen use & Coleoptera: Curculionidae & research & diagnostics \\
\hline 12-Oct & $\begin{array}{l}\text { Nikkola Hadley \& } \\
\text { friend }\end{array}$ & & & visit & & outreach & \\
\hline 12-Oct & Gary Peters & & $\begin{array}{l}\text { Copalis Beach, } \\
\text { WA }\end{array}$ & specimen use & Lepidoptera & & \\
\hline 12-Oct & Steve Vancampen & & & $\begin{array}{l}\text { education/ } \\
\text { workshop }\end{array}$ & Lepidoptera & & $\begin{array}{l}\text { NW } \\
\text { Lepidopterist } \\
\text { Workshop }\end{array}$ \\
\hline 14-Oct & Merrill Peterson & $\begin{array}{l}\text { Western } \\
\text { Washington } \\
\text { University }\end{array}$ & Bellingham, WA & $\begin{array}{l}\text { education/ } \\
\text { workshop }\end{array}$ & $\begin{array}{l}\text { Lepidoptera, Faunistic } \\
\text { coordination }\end{array}$ & $\begin{array}{l}\text { professional } \\
\text { connections }\end{array}$ & $\begin{array}{l}\text { NW } \\
\text { Lepidopterist } \\
\text { Workshop }\end{array}$ \\
\hline 14-Oct & $\begin{array}{l}\text { Jennifer Trehm \& } \\
\text { Joseph Smith }\end{array}$ & & & $\begin{array}{l}\text { education / } \\
\text { workshop }\end{array}$ & & $\begin{array}{l}\text { professional } \\
\text { connections }\end{array}$ & $\begin{array}{l}\text { NW } \\
\text { Lepidopterist } \\
\text { Workshop }\end{array}$ \\
\hline 23-Oct & NHCC club members & & OSU & visit & & outreach & \\
\hline 25-Oct & Ron Lyons & & Coos Bay, OR & specimen use & Hymenoptera:Anthidium & research & diagnostics \\
\hline 16-Nov & Jim Johnson & & Vancouver, WA & specimen use & Odonata & research & faunistics \\
\hline 20-Nov & Jessica Rerdon & & & & Hymenoptera: Halictidae & research & faunistics \\
\hline 20-Nov & Ron Lyons & & Coos Bay, OR & specimen use & $\begin{array}{l}\text { Coleoptera: } \\
\text { Chrysomelidae: } \\
\text { Galerucinae }\end{array}$ & research & faunistics \\
\hline 28-Nov & Alyssa Lee Suzumura & $\begin{array}{l}\text { Hokkaido } \\
\text { University }\end{array}$ & Hokkaido, Japan & specimen use & $\begin{array}{l}\text { Coleoptera: } \\
\text { Hydrophilidae: Cercyon I } \\
\text { Coleotpera: Salpingidae: } \\
\text { Aegialites I Coleoptera: } \\
\text { Tenebrionidae: } \\
\text { Phalaeromela }\end{array}$ & research & taxonomy \\
\hline
\end{tabular}




\begin{tabular}{|l|l|l|l|l|l|l|l|}
\hline date & name & affiliation & $\begin{array}{l}\text { city/state. } \\
\text { country }\end{array}$ & $\begin{array}{l}\text { purpose of } \\
\text { visit }\end{array}$ & taxon & visit category & research desc. \\
\hline 4-Dec & Peter Landolt & & & specimen use & $\begin{array}{l}\text { Coleoptera: Elateridae I } \\
\text { Hymenoptera: Sphecidae }\end{array}$ & $\begin{array}{l}\text { research \& } \\
\text { curation }\end{array}$ & research \\
\hline 7-Dec & Justin Runyon & & & specimen use & Diptera: Dolichopodidae & rese & research \\
\hline 11-Dec & Ron Lyons & & Coos Bay, OR & specimen use & $\begin{array}{l}\text { Coleoptera: } \\
\text { Chrysomelidae: } \\
\text { Galerucinae }\end{array}$ & faunistics \\
\hline 12-Dec & Claire Lonnsbury & & & & visit & & outreach \\
\hline 18-Dec & $\begin{array}{l}\text { Maureen Leong-Kee } \\
\text { \& Nicholas Martens }\end{array}$ & & OSU & visit & outreach & \\
\hline 20-Dec & Gregory S. Paulsen & & Corvallis, OR & visit & & & \\
\hline
\end{tabular}

